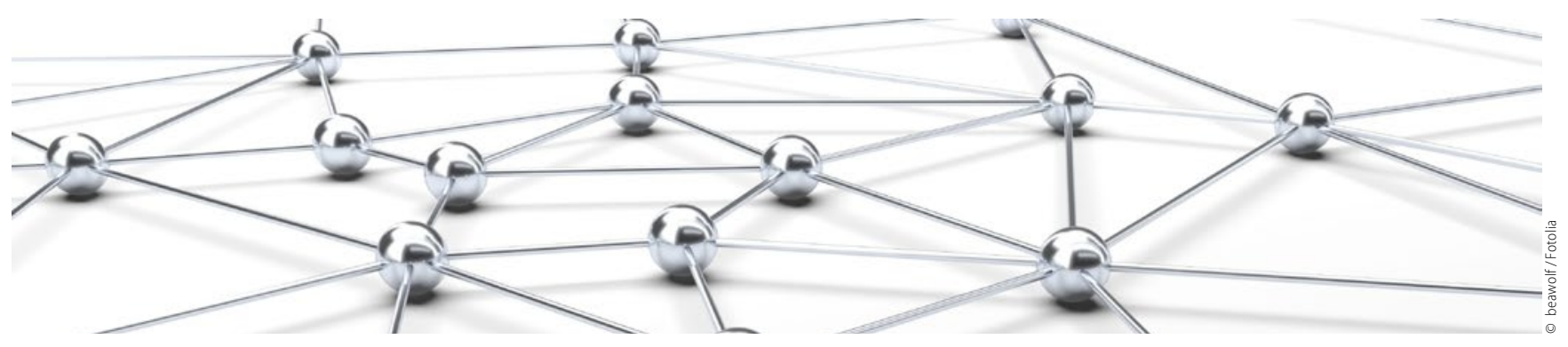

Knochenregeneration

\title{
Simultane Implantation und dreidimensional vorgeformte Titannetze
}

\begin{abstract}
Zur Augmentation des Alveolarknochens steht neben den sogenannten Knochenblöcken auch partikulärer Knochen oder Knochenersatzmaterial zur Verfügung. Dieses muss allerdings zur Rekonstruktion des Alveolarkamms stabilisiert werden, um einzuheilen. Hierfür eignen sich neben titanverstärkten Folien auch Titangitter.
\end{abstract}

Nachdem der Spenderknochen dreidimensional in die Form gefräst werden kann, die exakt in den Knochendefekt zur Rekonstruktion passt, stehen inzwischen auch dreidimensional vorgeformte Titangitter zur Verfügung. Zur Herstellung kommen Lasersinterungstechniken infrage, aber auch Anfertigungen von Titangittern an gedruckten Kiefermodellen des Patienten.

\section{Knochen bei Einheilung stabilisieren}

Bislang liegen nur wenige wissenschaftliche Arbeiten zu diesem Thema vor. In einer retrospektiven Studie untersuchten Lizio et. al. (2014) bei 15 alveolären Defekten den Verlust des augmentierten Knochens acht bis neun Monate, nachdem dieser mit Hilfe eines dreidimensional vorgeformten Gitters mit partikulärem Material aufgefüllt worden war. Die Volumina wurden mit Hilfe von Computertomografie-Aufnahmen evaluiert. Dabei konnte ein durchschnittlicher Knochenverlust von 30,2 Prozent festgestellt werden. Wunddehiszenzen traten in 80 Prozent der Fälle auf, im Mittel nach etwas mehr als zwei Monaten. Je größer die Wunddehiszenz war, desto größer war auch der Verlust an augmentiertem Kochenvolumen.

Eine andere Möglichkeit, die Knochenpartikel zur Einheilung zu stabilisieren, ist die simultane Implantation. Le et al. untersuchten die Augmentation buccaler Knochendefekte mit Hilfe von partikulärem, alloplastischem Knochenersatzmaterial bei transgingival einheilenden Implantaten unter Verwendung von Kollagenfolien. Dabei war die Augmentation vor allem bei kleinen und auch mittleren Knochendefekten $(<5 \mathrm{~mm})$ erfolgreich.

\section{Kurze Implantate bei Knochendefekten}

Esposito et al. haben im Rahmen einer Metaanalyse 13 randomisierte, kontrollierte klinische Studien untersucht und verschiedene Augmentationstechniken für sowohl horizontale als auch vertikale Knochendefekte untersucht. Zusammenfassend scheinen bei vertikalen Knochendefekten kurze Implantate die bessere Alternative zur Knochenaugmentation zu sein, da eine vertikale Augmentation häufig mit Komplikationen verbunden ist. Zur vertikalen Knochenregeneration scheint die Distraktionsosteogenese der Sandwichosteoplastik überlegen zu sein. Allogene Knochenersatzmaterialien scheinen dabei eine höhere Stabilität aufzuweisen. Zur horizontalen Knochenregeneration scheinen Auflagerungstechniken, zum Beispiel mit Hilfe von Knochenblöcken, geeignet zu sein, wobei die Fixierung per Titanschraube der Fixierung mit Hilfe resorbierbarer Materialien überlegen ist.

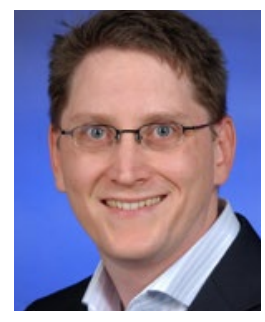

Prof. Dr. Dr. Felix P. Koch

Autor des Wissenschaftlichen Infodienstes, Lehrbeauftragter der Uniklinik Frankfurt, Praxisklinik für Mund-, Kiefer- und plastische Gesichtschirurgie in Wiesbaden. Mail:wid@fvdz.de 